\title{
METRIC SPACES AND POSITIVE DEFINITE FUNCTIONS*
}

\author{
BY \\ I. J. SCHOENBERG
}

1. Introduction. Let $E_{m}$ denote the $m$-dimensional euclidean space and generally $E_{m}{ }^{p}$ the pseudo-euclidean space of $m$ real variables with the distance function

$$
\left(\left|x_{1}-x_{1}^{\prime}\right|^{p}+\cdots+\left|x_{m}-x_{m}^{\prime}\right|^{p}\right)^{1 / p}, \quad \quad p>0 .
$$

As $p \rightarrow \infty$ we get the space $E_{m}^{\infty}$ with the distance function $\max _{i=1, \ldots, m}\left|x_{i}-x_{i}^{\prime}\right|$. Let, furthermore, $l^{p}$ stand for the space of real sequences with the series of $p$ th powers of the absolute values convergent. Similarly let $L^{p}$ denote the space of real measurable functions in the interval $(0,1)$ which are summable to the $p$ th power, while $C$ shall mean the space of real continuous functions in the same interval. In all these spaces a distance function is assumed to be defined as usual. $\dagger L^{2}$ is equivalent to the real Hilbert space $\mathfrak{S}$. The spaces $E_{m}^{p}, l^{p}$, and $L^{p}$ are metric only if $p \geqq 1$, but we shall consider them also for positive values of $p<1$. Finally, if $\subseteq$ is a (not necessarily metric) space with the distance function $\overline{P P^{\prime}}$, we shall denote by $\subseteq(\gamma)$ the new space which arises by changing the distance function from $\overline{P P}^{\prime}$ to $\overline{P P}^{\prime \gamma},(\gamma>0)$.

A general theorem of Banach and Mazur ([1], p. 187) states that any separable metric space $\subseteq$ may be imbedded isometrically in the space $C$. Furthermore, as a special case of a well known theorem of Urysohn, any such space $\mathfrak{S}$ may be imbedded topologically in $\mathfrak{S}$. Isometric imbeddability of $\mathfrak{S}$ in $\mathfrak{S}$ is, however, a much more restricted property of $\subseteq$.

The chief purpose of this paper is to point out the intimate relationship between the problem of isometric imbedding and the concept of positive definite functions, if this concept is properly enlarged. As a first approach to this connection we consider here isometric imbedding in Hilbert space only. It turns out that the possibility of imbedding $\ddagger$ in $\mathfrak{S}$ is very easily expressible in terms of the elementary function $e^{-t^{2}}$ and the concept of positive definite functions (Theorem 1). The author's previous result ([10]) to the effect that $\mathfrak{Y}(\gamma),(0<\gamma<1)$, which is the space arising from $\mathfrak{S}$ by raising its metric to a

* Presented to the Society, December 29, 1937; received by the editors December 14, 1937.

$\dagger$ See, for example, Banach [1], pp. 11-12. The numbers in square brackets refer to the list of references at the end of the paper.

$\ddagger$ Here and below the word "imbedding" stands for "isometrical imbedding." 
fractional power, is imbeddable in $\mathfrak{S},{ }^{*}$ appears again as a simple consequence (Corollary 1). For the class of spaces $\mathfrak{S}_{m}$ arising from the euclidean space $E_{m}$ by a general change of metric of the vector type (11) below, the condition of imbeddability in $\mathfrak{S}$ is directly expressible in a simple way in terms of the usual concept of positive definite functions as described by Mathias and Bochner (Theorem 2). The solution of this problem for $m=1$ (the problem of "screw lines" in $\mathfrak{H}$, von Neumann and Schoenberg [8]) allows us now to derive purely analytical results in the theory of positive definite functions with which it is equivalent. Two readily defined classes of positive definite functions are completely determined (Theorems 3 and 4). In particular two new proofs are given for the already known fact that the function $\exp \left[-|x|^{p}\right]$ is positive definite for values of $p$ in the range $0<p \leqq 2$, and not positive definite if $p>2 \uparrow(\$ 5)$. One proof is geometrical, the other proof covering the case $0<p \leqq 2$ is analytical and may be read independently of the rest of this paper.

All previous results now allow us to conclude that the spaces $E_{m}^{p}(\gamma), l^{p}(\gamma)$, and $L^{p}(\gamma)$ are imbeddable in $\mathfrak{S}$ for values of $\gamma$ in the range $0<\gamma \leqq p / 2$, where $p$ is restricted to the range $0<p \leqq 2$ (Theorem 5 ). For $p=2$ we regain our previous result concerning $\mathfrak{S}(\gamma)$. It is interesting to compare and combine this result about $L^{p}$ with a theoem of Banach and Mazur ([1], p. 203) concerning the linear dimensions of $L^{2}$ and $L^{q}$. According to this theorem $L^{2}$ is imbeddable in $L^{q}$ if $q>1$. Now as $L^{p}(\gamma),(0<p \leqq 2,0<\gamma \leqq p / 2)$, is imbeddable in $\mathfrak{S}$ or $L^{2}$, it follows that $L^{p}(\gamma),(0<p \leqq 2,0<\gamma \leqq p / 2)$, is imbeddable in any $L^{q}$ if $q>1$.

Similar as yet unsolved problems concerning the case of $p>2$ are shown to be equivalent to further knowledge as to the positive definite character of certain special functions of $m$ variables. One of these unsolved problems suggests a probably possible way of extending an interesting theorem of L. M. Blumenthal on metric sets of four points to such sets of any number of points.

The reader primarily interested in the geometrical results of this paper may omit $\$ 4$ and $\$ 5$ entirely by taking the known Corollary 3 for granted.

2. Positive definite functions. A real continuous function $f\left(x_{1}, x_{2}, \cdots, x_{m}\right)$ which is defined for all real values of its variables and is even, that is for which $f\left(-x_{1}, \cdots,-x_{m}\right)=f\left(x_{1}, \cdots, x_{m}\right)$, is said to be positive definite (p.d.) if

$$
\sum_{i, k=1}^{n} f\left(x_{1}^{(i)}-x_{1}^{(k)}, \cdots, x_{m}^{(i)}-x_{m}^{(k)}\right) \rho_{i} \rho_{k} \geqq 0
$$

* W. A. Wilson [12], had previously remarked that $E_{1}(1 / 2)$ is imbeddable in $\mathfrak{S}$.

$\dagger$ Due in various parts to Pólya, Mathias, and P. Lévy. For references see Bochner [3], pp. 76-77. For still another treatment of the case $0<p \leqq 2$ see Bochner [5]. 
for arbitrary real $\rho_{i}$ and any $n$ points $\left(x^{(i)}\right),(i=1, \cdots, n)$, for $n=2,3, \cdots$. For $n=2,(1)$ gives $\left|f\left(x_{1}, \cdots, x_{m}\right)\right| \leqq f(0, \cdots, 0)$, hence p.d. functions are bounded throughout space and take their maximum value at the origin. We shall use in the sequel only the following most simple properties of this interesting class of functions.

I. The function defined by

$$
\begin{aligned}
f\left(x_{1}, \cdots, x_{m}\right)=\int_{-\infty}^{\infty} \cdots & \int_{-\infty}^{\infty} \psi\left(u_{1}, \cdots, u_{m}\right) \\
& \cdot \exp \left[i\left(x_{1} u_{1}+\cdots+x_{m} u_{m}\right)\right] d u_{1} \cdots d u_{m},
\end{aligned}
$$

where $\psi$ is a non-negative even function such that its integral over the whole space exists, is p. d.

II. Any finite linear combination of p.d. functions with non-negative coefficients is again p.d. The product of two p.d. functions is again p.d.

III. A continuous function which is the limit of a sequence of p.d. functions is itself p.d.

For completeness we sketch the simple proofs. Property I follows from the fact that the left-hand side of (1) reduces to

$$
\int \cdots \int\left|\sum_{k=1}^{n} \rho_{k} \exp \left[i\left(x_{1}^{(k)} u_{1}+\cdots+x_{m}^{(k)} u_{m}\right)\right]\right|^{2} \psi d u_{1} \cdots d u_{m} \geqq 0 .
$$

The additive property is clear from the fact that (1) is a linear inequality in $f$. The multiplicative property is a direct consequence of a lemma of I. Schur ([11],p. 10)* which states that if $\sum_{1}^{n} a_{i k} \rho_{i} \rho_{k}, \sum_{1}^{n} b_{i k} \rho_{i} \rho_{k}$, are two positive quadratic forms, then $\sum_{1}^{n} a_{i k} b_{i k} \rho_{i} \rho_{k}$ is also positive. Property III is immediately clear by continuity. We shall later on use the fact that $f(x)=\cos \lambda x$ is a p.d. function.

A closely allied concept is as follows. Let $\subseteq$ be a space in which a distance function $\overline{P P^{\prime}}$ is defined subject to the following conditions: (1) $\overline{P P^{\prime}}=\overline{P^{\prime} P} \geqq 0$ for arbitrary points $P, P^{\prime}$ in $\Im$, (2) $\overline{P P}=0$. A real continuous even function $g(t)$, which is defined in the range of values of $\pm \overline{P P}^{\prime},\left(P, P^{\prime}\right.$ in $\left.\Im\right)$, is said to be positive definite in $\subseteq$ if

$$
\sum_{i, k=1}^{n} g\left(\overline{P_{i} P_{k}}\right) \rho_{i} \rho_{k} \geqq 0
$$

for arbitrary real $\rho_{i}$ and any $n$ points $P_{i}$ (different or not) of $\subseteq,(n=2,3, \cdots)$.

* For a discussion and consequences of Schur's result see also Pólya and Szegö [9], pp. 106-107, 307-308. 
This class of functions $g(t)$ has for a given space $\subseteq$ properties II and III above for similar reasons. Both definitions agree if $m=1$, while $\subseteq$ is the one-dimensional euclidean space $E_{1}$.

The peculiar relationship between the definitions, which will be clearer later on, is already exhibited by the following simple example needed in the sequel. From the formula

$$
e^{-x^{2}}=2^{-1} \pi^{-1 / 2} \int_{-\infty}^{\infty} e^{i x u} e^{-u^{2} / 4} d u
$$

we get, replacing $x$ by $x_{j},(j=1, \cdots, m)$, and multiplying the resulting equations,

$$
\begin{aligned}
& \exp \left[-\left(x_{1}^{2}+\cdots+x_{m}{ }^{2}\right)\right]=\exp \left[-\left[\left(x_{1}^{2}+\cdots+x_{m}{ }^{2}\right)^{1 / 2}\right]^{2}\right] \\
&=2^{-m} \pi^{-m / 2} \int_{-\infty}^{\infty} \cdots \int_{-\infty}^{\infty} \exp \left[i\left(x_{1} u_{1}+\cdots+x_{m} u_{m}\right)\right] \\
& \cdot \exp \left[-\left(u_{1}^{2}+\cdots+u_{m}{ }^{2}\right) / 4\right] d u_{1} \cdots d u_{m}
\end{aligned}
$$

which shows at a glance (property I) that the function

$$
f=\exp \left[-\left(x_{1}^{2}+\cdots+x_{m}^{2}\right)\right]
$$

is p.d. and also that $g(t)=e^{-t^{2}}$ is p.d. in $E_{m}$. As $m$ is arbitrary, this implies that the function $e^{-t^{2}}$ is positive definite in the real Hilbert space $\mathfrak{S}$.

3. Conditions for isometric imbedding in Hilbert space in terms of positive definite functions. It was pointed out by $\mathrm{K}$. Menger and by the author (for references see [10]) that a necessary and sufficient condition that a separable space $\mathfrak{\subseteq}$ be imbeddable in $\mathfrak{S}$ is that for any $n+1$ points of $\mathfrak{S},(n \geqq 2)$, we have

$$
\sum_{i, k=1}^{n}\left(\overline{P_{0} P_{i}^{2}}+\overline{P_{0} P_{k}^{2}}-{\overline{P_{i} P_{k}}}^{2}\right) \rho_{i} \rho_{k} \geqq 0,
$$

for arbitrary real $\rho_{i} *$ Let us now put this condition in a slightly more symmetrical form. By summing over the three terms separately, we may write this as

$$
2 \sum_{1}^{n} \rho_{k} \cdot \sum_{1}^{n}{\overline{P_{0} P_{k}}}^{2} \rho_{k}-\sum_{1}^{n}{\overline{P_{i} P_{k}}}^{2} \rho_{i} \rho_{k} \geqq 0,
$$

* This was proved for the case when $\subseteq$ is a separable semi-metric space; that is, when the metric $\overline{P P}^{\prime}$ satisfies the additional condition (3) $\overline{P P}^{\prime}>0$ if $P \neq P^{\prime}$, whereas we postulated only that (1) $\overline{P P}^{\prime}=\overline{P^{\prime} P} \geqq 0$, (2) $\overline{P P}=0$. However, our quadratic inequality, for $n=2$, insures the triangle inequality $\overline{P Q}+\overline{Q R} \geqq \overline{P R}$ for any three points of $\Im$. If we now identify with $P$ all points $Q$ such that $\overline{P Q}=0$ (which is now allowed, since $\overline{P Q}=0$ implies $\overline{R P}=\overline{R Q}$ for any $R$, on account of the triangle inequality) and do this for all points of $\Xi$, we get a new space which is not only semi-metric but even metric. 
and if we set $\rho_{0}=-\sum_{1}^{n} \rho_{k}$, this last inequality is equivalent to

or, finally,

$$
-\rho_{0}^{2}{\overline{P_{0} P_{0}^{2}}}^{2}-2 \sum_{0}^{n}{\overline{P_{0} P_{k}}}^{2} \rho_{0} \rho_{k}-\sum_{1}^{n}{\overline{P_{i} P_{k}^{2}}}^{2} \rho_{i} \rho_{k} \geqq 0,
$$

$$
\sum_{i, k=0}^{n} \overline{P_{i} P_{k}^{2}} \rho_{i} \rho_{k} \leqq 0 .
$$

Hence the inequality (5), as a consequence of the relation

$$
\sum_{i=0}^{n} \rho_{i}=0,
$$

is equivalent to the above stated condition of imbeddability.

Now we are prepared to express this condition in terms of p.d. functions. We have seen in the previous section that the function $e^{-t^{2}}$ is p.d. in $\mathfrak{S}$. Clearly also $e^{-\lambda^{2} t^{2}}$ ( $\lambda$ real) is p.d. in $\mathfrak{S}$ as can be seen from (4) or by direct reasoning. Hence $e^{-\lambda^{2} t^{2}}$ is also p.d. in any subset of $\mathfrak{S}$. Now if $\subseteq$ is to be imbeddable in $\mathfrak{S}$, it is clearly a necessary condition that $e^{-\lambda^{2} t^{2}}$ be p.d. in $\mathfrak{S}$.

Let us prove now that this condition, together with the separability of $\mathfrak{S}$, is also sufficient. To prove this we have to show that (5) holds as a consequence of (6) for any $n+1$ points $P_{i}$ of $\mathfrak{S}$. Now as $e^{-\lambda^{2} t^{2}}$ is p.d. in $\mathfrak{S}$, we have

$$
\sum_{0}^{n} \rho_{i} \rho_{k} \exp \left[-\lambda^{2} \overline{P_{i} P_{k}^{2}}\right] \geqq 0
$$

by (3). We complete the proof in two different ways.

First proof. By expanding the left-hand side of (7) in power series we have, in view of (6),

$$
-\lambda^{2}\left(\sum_{0}^{n} \overline{P_{i} P_{k}^{2}} \rho_{i} \rho_{k}\right)+\lambda^{4}\left(\sum_{0}^{n} \overline{P_{i} P_{k}^{4}} \rho_{i} \rho_{k}\right) / 2-\cdots \geqq 0,
$$

which clearly implies (5) for small values of $\lambda$.

Second proof. Using the formulas

$$
\begin{aligned}
t^{\alpha} & =c(\alpha) \int_{0}^{\infty}\left(1-e^{-\lambda^{2} t^{2}}\right) \lambda^{-1-\alpha} d \lambda, \\
c(\alpha) & =\left[\int_{0}^{\infty}\left(1-e^{-\lambda^{2}}\right) \lambda^{-1-\alpha} d \lambda\right]^{-1}, \quad 0<\alpha<2, t \geqq 0,
\end{aligned}
$$

which are immediately proved by substituting in the first integral $\lambda t^{-1}$ for $\lambda$, we have 


$$
\overline{P_{i} P_{k}^{\alpha}}=c(\alpha) \int_{0}^{\infty}\left(1-\exp \left[-\lambda^{2}{\overline{P_{i} P_{k}}}^{2}\right]\right) \lambda^{-1-\alpha} d \lambda .
$$

If now $\rho_{i}$ are any real numbers satisfying (6), we get, for $0<\alpha<2$,

$$
\sum_{i, k=0}^{n} \overline{P_{i} P_{k}^{\alpha} \rho_{i} \rho_{k}}=-c(\alpha) \int_{0}^{\infty}\left\{\sum_{0}^{n} \rho_{i} \rho_{k} \exp \left[-\lambda^{2} \overline{P_{i} P_{k}^{2}}\right]\right\} \lambda^{-1-\alpha} d \lambda \leqq 0,
$$

by (7) and the obvious fact that $c(\alpha)>0$. Now we get again the desired inequality (5) on allowing $\alpha$ in (9) to approach the limit 2. We have thus proved the following theorem:

THEOREM 1. A necessary and sufficient condition that a separable space $\mathfrak{S}$ with a distance function $\overline{P P^{\prime}}$ with the properties $\overline{P P^{\prime}}=\overline{P^{\prime} P} \geqq 0, \overline{P P}=0$, be isometrically imbeddable in $\mathfrak{S}$, is that the family of functions $e^{-\lambda t^{2}},(\lambda>0)$, be positive definite in S.

Notice that the condition of this theorem may be restricted to require that $e^{-\lambda t^{2}}$ be p.d. in $\subseteq$ only for a set of positive values of $\lambda$ admitting the origin $\lambda=0$ as a point of accumulation. The properties II and III ( $\$ 2)$ will then imply that $e^{-\lambda t^{2}}$ is p.d. in $\subseteq$ for all positive values of $\lambda$.

Recalling that we denote by $\mathfrak{S}(\gamma),(\gamma>0)$, the space obtained from $\mathfrak{S}$ by replacing its metric $\widehat{P P}^{\prime}$ by $\overrightarrow{P P}^{\prime \gamma}$, it is of interest to point out the further fact, implicitly contained in the previous second proof, that if $\subseteq$ is imbeddable in $\mathfrak{S}$, then so is $\mathfrak{S}(\gamma)$ for any value of $\gamma$ in the range $0<\gamma<1$. Indeed, let $\alpha=2 \gamma$; if $\mathfrak{S}$ is imbeddable in $\mathfrak{S}$, then $e^{-\lambda t^{2}},(\lambda>0)$, is p. d. in $\mathfrak{S}$; hence (9) holds in virtue of $(6)$, and $\subseteq(\gamma)$ is therefore imbeddable in $\mathfrak{S}$ on account of the form (5) of the imbeddability condition. Applying this conclusion to $\mathfrak{S}=\mathfrak{S}$ itself, we have the following corollary:

COROLlaRy 1. The space $\mathfrak{S}(\gamma),(0<\gamma<1)$, obtained from Hilbert space $\mathfrak{S}$ by raising its metric to a power $\gamma$, is imbeddable in $\mathfrak{S}_{.}^{*}$

* We may even state the following more general theorem: Let

$$
F(t)=\int_{0}^{\infty}\left(1-e^{-\lambda 2 t 2}\right) \lambda^{-2} d \sigma(\lambda),
$$

where $\sigma(\lambda)$ is non-decreasing for $\lambda \geqq 0$ and is such that $\int_{1}^{\infty} \lambda^{-2} d \sigma(\lambda)$ exists. If we change the metric of $\mathfrak{g}$ from $\overline{P P^{\prime}}$ to $\left[F\left(\overline{P P}^{\prime}\right)\right]^{1 / 2}$, then the new space thus arising is imbeddable in $\mathfrak{\mathbb { E }}$. Indeed $(6),(7)$, and $\left(8^{\prime}\right)$ imply

$$
\sum_{1}^{n} F\left(\overline{P_{i} P_{k}}\right) \rho_{i} \rho_{k}=-\int_{0}^{\infty}\left(\sum_{i, k=1}^{n} \rho_{i} \rho_{k} \exp \left[-\lambda^{2}{\overline{P_{i} P_{k}}}^{2}\right]\right) \lambda^{-2} d \sigma(\lambda) \leqq 0,
$$

and the theorem follows on account of the form (5) and (6) of the imbeddability condition. We leave open the question whether or not $\left(8^{\prime}\right)$ gives the most general function $F(t)$ with this property.

Added in proof, August, 1938: Formula $\left(8^{\prime}\right)$ gives indeed all functions with the property stated above. See the following paper Metric spaces and completely monotone functions, to appear in the Annals of Mathematics. 
We now turn our attention to a special class of spaces of the type $₫$ which are defined as follows. Let $\phi\left(x_{1}, \cdots, x_{m}\right)$ be a continuous function defined for all real values of its variables with the following properties

$$
\begin{gathered}
\phi\left(x_{1}, \cdots, x_{m}\right) \geqq 0, \quad \phi(0, \cdots, 0)=0, \\
\phi\left(-x_{1}, \cdots,-x_{m}\right)=\phi\left(x_{1}, \cdots, x_{m}\right) ;
\end{gathered}
$$

and call $\mathfrak{S}_{m}$ the space of points $P=\left(x_{1}, \cdots, x_{m}\right)$ with the distance function

$$
\overline{P P}^{\prime}=\left[\phi\left(x_{1}-x_{1}^{\prime}, \cdots, x_{m}-x_{m}^{\prime}\right)\right]^{1 / 2} .
$$

For such a space, which is obviously separable, the condition of Theorem 1 that $e^{-\lambda t^{2}}$ be p.d. in $\Im_{m}$ may be expressed in a more familiar form. Indeed, on comparing the formulas (1), (3), and (11), we now obtain from Theorem 1 the following theorem:

THEOREM 2. The space $\mathfrak{S}_{m}$ of $m$ real numbers with the metric (11) is imbeddable in $\mathfrak{S}$ if and only if the functions

$$
f_{\lambda}=\exp \left[-\lambda \phi\left(x_{1}, \cdots, x_{m}\right)\right], \quad \lambda>0,
$$

are positive definite in the sense (1) of Mathias and Bochner for all positive values of $\lambda$.

If $\phi\left(x_{1}, \cdots, x_{m}\right)$ is a homogeneous function, ${ }^{*}$ the conditions (12) may be replaced by the single condition that the function

$$
f_{1}=\exp \left[-\phi\left(x_{1}, \cdots, x_{m}\right)\right]
$$

be positive definite.

We mention the following corollary:

Corollary 2. If $\phi\left(x_{1}, \cdots, x_{m}\right)$ is homogeneous and such that $e^{-\phi}$ is positive definite, then

$$
e^{-\phi^{\gamma}}, \quad 0<\gamma<1 \text {, }
$$

is also positive definite.

For if $e^{-\phi}$ is p.d., then by Theorem $2, \mathfrak{\subseteq}_{m}$ is imbeddable in $\mathfrak{S}$ and therefor $\mathfrak{S}_{m}(\gamma)$ is also (Corollary 1 ). Hence $\exp \left[-\phi^{\gamma}\right]$ is seen to be p.d. by applying Theorem 2 the other way around.

4. Determination of certain classes of positive definite functions. In this section we shall assume $m=1, \phi(x)$ being therefore a continuous non-negative even function vanishing at the origin. In this case we know precisely when $\mathfrak{\subseteq}_{1}$,

* We say that $\phi$ is homogeneous of degree $\kappa$ if $\phi\left(t x_{1}, \cdots, t x_{m}\right)=t^{\kappa} \phi\left(x_{1}, \cdots, x_{m}\right)$ holds identically in the $x_{i}$ and for $t>0$. A continuous homogeneous function $\phi$ with the properties (10) must, unless it vanishes identically, have a positive degree $\kappa$. 
which is the space of real numbers with the metric $\left[\phi\left(x-x^{\prime}\right)\right]^{1 / 2}$ is imbeddable in $\mathfrak{S}$ (von Neumann and Schoenberg [8]). The most general function $\phi(x)$ for which this is the case is of the form

$$
\phi(x)=\int_{0}^{\infty} \frac{\sin ^{2} x u}{u^{2}} d \sigma(u),
$$

where $\sigma(u)$ is non-decreasing for $u \geqq 0$ such that

$$
\int_{1}^{\infty} \frac{d \sigma(u)}{u^{2}} \text { exists. }
$$

Hence by Theorem 2 we infer that if

$$
[f(x)]^{\lambda}=e^{-\lambda \phi(x)}
$$

is p.d. for $\lambda>0$, then $\phi(x)$ is of the form (14) and conversely. If now $f(x)$ is any positive function whose positive powers $[f(x)]^{\lambda}$ are all p.d., then considering

$$
f(x) / f(0)=e^{-\phi(x)},
$$

where $\phi(x)$ is necessarily non-negative, even, and vanishing at the origin, we conclude as before that $\phi(x)$ is of the form (14). We have thus proved the following theorem:

THEOREM 3. The most general positive function $f(x)$ whose positive powers $[f(x)]^{\lambda},(\lambda>0)$, are all positive definite is of the form

$$
f(x)=\exp \left\{c-\int_{0}^{\infty} \frac{\sin ^{2} x u}{u^{2}} d \sigma(u)\right\},
$$

where $\sigma(u)$ is a non-decreasing function subject to the restriction (15), while $c$ is any real constant.

A few remarks are called for regarding the condition of this theorem that $[f(x)]^{\lambda}$ be p.d. for $\lambda>0$. In the first place, as remarked after the statement of Theorem 1, the range of $\lambda$ in this condition may be restricted to a sequence of positive numbers tending to zero.

A second and more important remark is that Theorem 3 becomes false if we assume only that the positive function $f(x)$ is p.d., or in other words: formula (16) always represents a positive and p.d. function; it does not, however, represent all such functions but only those whose fractional powers are also p. d. To prove this statement it suffices to exhibit a p.d. function $f(x)>0$ such that $[f(x)]^{\lambda},(0<\lambda<1)$, are not all p.d. Such a function is

$$
f_{\epsilon}(x)=\epsilon^{2}+\cos ^{2} x
$$


if $\epsilon$ is sufficiently small, for it is easy to see that

$$
\left[f_{\epsilon}(x)\right]^{1 / 2}=\left(\epsilon^{2}+\cos ^{2} x\right)^{1 / 2}
$$

is not p.d. for such values of $\epsilon$. Indeed, if it were p.d. for arbitrarily small $\epsilon$, it would follow that the limiting function

$$
\lim _{\epsilon \rightarrow 0}\left[f_{\epsilon}(x)\right]^{1 / 2}=|\cos x|
$$

were also p.d. (property III). But this is not the case as is seen from the fact that the cosine series

$$
|\cos x|=\frac{2}{\pi}+\frac{4}{\pi} \sum_{n=1}^{\infty}(-1)^{n-1} \frac{\cos 2 n x}{4 n^{2}-1}
$$

has some negative coefficients, or even more directly from the fact that the form

$$
\sum_{i, k=1}^{4}\left|\cos \left(x_{i}-x_{k}\right)\right| \rho_{i} \rho_{k}
$$

where $x_{1}=0, x_{2}=\pi / 4, x_{3}=\pi / 2, x_{4}=3 \pi / 4$, is not positive. Indeed, its determinant is readily found to be -1 , hence negative. The essence of the matter is that Schur's theorem, "If $\sum a_{i k} \rho_{i} \rho_{k}$ is positive, then so is $\sum a_{i k}^{n} \rho_{i} \rho_{k}$ for $n=1,2,3, \ldots$, , can not be so extended that we may conclude that $\sum\left|a_{i k}\right|{ }^{\lambda} \rho_{i} \rho_{k},(\lambda>0)$, is positive.

The third and last remark is that Theorem 3 is now equivalent to the theorem that (14) and (15) give the most general $\phi(x)$ such that the space of real numbers with the metric $\left[\phi\left(x-x^{\prime}\right)\right]^{1 / 2}$ be imbeddable in $\mathfrak{S}$ (see [8]). Hence a direct proof of Theorem 3 would furnish a new proof of that theorem.

While the problem of determining all positive and positive definite functions is yet unsolved, there is another subclass of this class of functions which can now be readily determined. It will in fact be a subclass of the class determined by Theorem 3 .

Let $\psi(x)$ be a p.d. function, $c$ a real constant. Clearly

$$
f(x)=\exp [c+\psi(x)]=e^{c}\left(1+\frac{1}{1 !} \psi(x)+\frac{1}{2 !} \psi^{2}(x)+\cdots\right)
$$

is also p.d. in virtue of the properties II and III. It has moreover the following additional two properties: $(\alpha)$ It is bounded away from zero, since $f(x) \geqq \exp [c-\psi(0)]>0$. ( $\beta)$ All its positive powers $[f(x)]^{\lambda},(\lambda>0)$, are also p.d. Let us show that the converse is true, that any function $f(x)$ having the properties $(\alpha)$ and $(\beta)$ is of the form (17) where $\psi(x)$ is p.d. Now an $f(x)$ with the properties $(\alpha)$ and $(\beta)$ clearly belongs to the class described by Theo- 
rem 3, and all we have to do is to decide which functions of the form (16) are bounded away from zero. Now this is the case if and only if the exponent (14), namely

$$
\phi(x)=\int_{0}^{\infty} \frac{\sin ^{2} x u}{u^{2}} d \sigma(u)
$$

is bounded in $-\infty<x<\infty$. The characteristic conditions for this have likewise been determined by von Neumann and the author in [8] and they are as follows: $\phi(x)$ is bounded if and only if $\sigma(0)=\sigma(+0)$ and $\int_{+0}^{\infty} u^{-2} d \sigma(u)$ exists. But if these conditions are fulfilled, then the non-decreasing function

$$
\tau(u)=\int_{+0}^{u} \frac{d \sigma(u)}{u^{2}}
$$

is bounded for $u>0$, and (14) can be written as

$$
\begin{aligned}
\phi(x) & =\int_{+0}^{\infty} \frac{\sin ^{2} x u}{u^{2}} d \sigma(u)=\int_{+0}^{\infty} \sin ^{2} x u d \tau(u) \\
& =\frac{1}{2} \int_{+0}^{\infty}(1-\cos 2 x u) d \tau(u)=\psi(0)-\psi(x),
\end{aligned}
$$

where

$$
\psi(x)=\frac{1}{2} \int_{+0}^{\infty} \cos 2 u x d \tau(u)
$$

is p.d. Hence, by (14), (18), and (16),

$$
f(x)=\exp [c-\phi(x)]=\exp [c-\psi(0)+\psi(x)]
$$

and is indeed of the desired form (17). We have thus proved the following theorem:

THEOREM 4. The most general positive function $f(x)$ which is bounded away from zero and whose positive powers $[f(x)]^{\lambda},(\lambda>0)$, are positive definite is of the form

$$
f(x)=\exp [c+\psi(x)]
$$

where $\psi(x)$ is positive definite and $c$ is a real constant.

Equivalent Statement: If $f(x)$ is a positive function, then $\log f(x)$ differs by a constant from a positive definite function if and only if $f(x)$ is bounded away from zero and its powers $[f(x)]^{\lambda},(\lambda>0)$, are all positive definite.

5. On the positive definite character of $\exp \left[-|x|^{p}\right]$. We shall need in the next section the following well known result: 
Corollary 3. The function

$$
\exp \left[-|x|^{p}\right]
$$

is positive definite if $0<p \leqq 2$ and not positive definite if $p>2$.

First proof. We know that exp $\left[-x^{2}\right]$ is p.d. from the formula just preceding (4) and property I. Hence exp [ $\left.-|x|^{2 \gamma}\right],(0<\gamma<1)$, is p.d. by Corollary 2 , as $\phi(x)=|x|^{2}$ is homogeneous, and the first part of the statement is proved. Suppose now that (19) were p.d. for a value $p>2$. By Theorem 2 this would imply that the space $E_{1}(p / 2)$, which is the real axis with the metric $\left|x-x^{\prime}\right| p / 2$, were imbeddable in $\mathfrak{S}$. But this is clearly impossible as $E_{1}(p / 2)$ is not even a metric space since the distances among the points $x=0,1,2$ are $\overline{01}=1, \overline{12}=1$, $\overline{02}=2^{p / 2}>2$, and they violate the triangle inequality.

Second direct proof for the case $0<p \leqq 2$. First we shall prove directly on the basis of properties II and III ( $\$ 2)$ that the function $f(x)$ defined by (16) and (15) is p.d. By property III $(T \rightarrow \infty)$ it suffices to show that

$$
\exp \left[-\int_{0}^{T} \frac{\sin ^{2} x u}{u^{2}} d \sigma(u)\right]
$$

is p.d. By the definition of the Stieltjes integral, this is a limit of functions of the form

$$
\exp \left[-\sum_{\nu=1}^{n} 2 A_{\nu} \sin ^{2} x u_{\nu}\right], \quad \quad A_{\nu}>0,
$$

and it suffices to show that each of the factors is p.d. Now

$$
\exp \left[-2 A \sin ^{2} x u\right]=\exp [-A] \exp [A \cos 2 x u],
$$

and this is p.d. because $\cos 2 x u$ is p.d. and the exponential series has positive coefficients only. This point being disposed of, it suffices to show that $|x|^{p}$, $(0<p \leqq 2)$, is of the form (14).* This is apparent on account of the formula

$$
|x|^{p}=\int_{0}^{\infty} \sin ^{2} x u \cdot u^{-1-p} d u / \int_{0}^{\infty} \sin ^{2} u \cdot u^{-1-p} d u,
$$

valid for $0<p<2$, which is as easily established as the similar representation (8). An obvious step-function for $\sigma(u)$ in (14), with only one jump at the origin, settles the case $p=2$.

6. On the imbedding of the spaces $E_{m}^{p}, l^{p}, L^{p},(0<p \leqq 2)$, in $\mathfrak{S}$ by a change of metric. We learned in $\$ 2$ that $e^{-t^{2}}$ is p.d. in $\mathfrak{S}$ or $L^{2}$. We shall now show

\footnotetext{
* It is not by accident that the function (19) is of the form (16) if $0<p \leqq 2$. For if (19) is p.d., its positive powers must also be p.d., and the function is necessarily of the form (16), by Theorem 3 . Notice that it is not bounded away from zero and hence is not of the form (17).
} 
that $e^{-|t|^{p}}$ is p.d. in the spaces $E_{m}^{p}, l^{p}$, and $L^{p}$ for values of $p$ in the range $0<p \leqq 2$.

The function $\exp \left[-|x|^{p}\right]$ was found to be p.d. if $0<p \leqq 2$. Hence the functions $\exp \left[-\left|x_{i}\right|^{p}\right],(i=1,2, \cdots, m)$, are also p.d. when regarded as functions of the $m$ variables $x_{i}$, and we may infer, by property II $(\$ 2)$, that their product

$$
f=\exp \left[-\left(\left|x_{1}\right|^{p}+\cdots+\left|x_{m}\right|^{p}\right)\right], \quad 0<p \leqq 2,
$$

is p.d. But this is equivalent to the statement that exp $\left[-|t|^{p}\right]$ is p.d. in $E_{m}{ }^{p}$. As the similar statement for $l^{p}$ is proved in the same way as for $L^{p}$, only requiring less care, we shall limit ourselves to the consideration of $L^{p}$. Let

$$
P_{i}=x_{i}(t) \text {, }
$$

where

$$
\int_{0}^{1}\left|x_{i}(t)\right|^{p} d t, \quad i=1,2, \cdots, n
$$

exists, be $n$ points of $L^{p}$. For

$$
\overline{P_{i} P_{k}}=\left(\int_{0}^{1}\left|x_{i}(t)-x_{k}(t)\right|^{p} d t\right)^{1 / p}
$$

we have to show that

$$
\sum_{i, k=1}^{n} \rho_{i} \rho_{k} \exp \left[-{\overline{P_{i} P_{k}^{p}}}^{p}\right] \geqq 0
$$

for real $\rho_{i}$. There is no loss of generality in assuming that the functions $x_{i}(t)$ are continuous, as continuous functions are everywhere dense in $L^{p}$. For

$$
x_{\nu i}=m^{-1 / p} \cdot x_{i}(\nu / m), \quad \nu=1,2, \cdots, m ; i=1,2, \cdots, n,
$$

we have

$$
\begin{aligned}
\left(\sum_{\nu=1}^{m}\left|x_{\nu i}-x_{\nu k}\right|^{p}\right)^{1 / p} & =\left(\frac{1}{m} \sum_{\nu=1}^{m}\left|x_{i}(\nu / m)-x_{k}(\nu / m)\right|^{p}\right)^{1 / p} \\
& \rightarrow \overline{P_{i} P_{k}}, \quad m \rightarrow \infty,
\end{aligned}
$$

which proves the inequality (20), for (20) is already known to hold for all values of $m$ if the $\overline{P_{i} P_{k}}$ of $(20)$ are replaced by the quantities on the left-hand side of (21).

The fact that $\exp \left[-|t|^{p}\right],(0<p \leqq 2)$, is p.d. in $E_{m}^{p}, l^{p}, L^{p}$ implies that $\exp \left[-|t|^{2}\right]$, and therefore also exp $\left[-\lambda|t|^{2}\right],(\lambda>0)$, is p.d. in $E_{m}^{p}(p / 2)$, $l^{p}(p / 2)$, and $L^{p}(p / 2)$. We have thus proved the following theorem: 
THEOREM 5. The spaces $E_{m}{ }^{p}, l^{p}$, and $L^{p},(0<p \leqq 2)$, become imbeddable in $\mathfrak{S}$ if we raise their metrics to the power $p / 2$ or less. In other words, $E_{m}{ }^{p}(\gamma), l^{p}(\gamma)$, and $L^{p}(\gamma)$ are imbeddable in $\mathfrak{S}$ for values of $\gamma$ in the range $0<\gamma \leqq p / 2$. $^{*}$

Note that for $p=2$ this result coincides with Corollary 1 .

The fact that $E_{m}{ }^{p},(0<p<1)$, which is not a metric space, may be made metric by raising its metric to a suitable power was already known in a different terminology. Indeed, the following readily established "substitute" for Minkowski's inequality ([7], p. 32)

$$
\sum_{1}^{m}\left|x_{i}+y_{i}\right|^{p} \leqq \sum_{1}^{m}\left|x_{i}\right|^{p}+\sum_{1}^{m}\left|y_{i}\right|^{p}, \quad 0<p<1,
$$

means precisely that $E_{m} p(p)$, and therefore $E_{m} p(\gamma),(0<\gamma \leqq p)$, is a metric space. Now Theorem 5 states that if we restrict this range of $\gamma$ to $0<\gamma \leqq p / 2$, then not only can any three points of $E_{m} p(\gamma)$ be imbedded isometrically in a euclidean space, but the same is true for any number of such points, since the whole space is imbeddable in Hilbert space.

7. Some unsolved problems. We shall devote this last section to a few unsolved analytical problems and to a brief discussion of their geometrical implications.

Problem 1. Let $p$ be a real number exceeding 2 , and let $m=2,3, \cdots$. Do there exist positive exponents $\kappa$ such that the function

$$
\exp \left[-\left(\left|x_{1}\right|^{p}+\cdots+\left|x_{m}\right|^{p}\right)^{\kappa}\right]
$$

is positive definite?

If there are such positive exponents, then Corollary 2 implies the existence of a positive number $\kappa_{m}$ with the properties: (22) is p.d. if $0<\kappa \leqq \kappa_{m}$ and is not p.d. if $\kappa>\kappa_{m}$. Furthermore Corollary 3 implies that $p \kappa_{m} \leqq 2$ or $\kappa_{m} \leqq 2 / p$. Theorem 2 furnishes the following geometrical equivalent to Problem 1 . The function (22) is p.d. if and only if $E_{m}^{p}(p \kappa / 2)$, and therefore also $l^{p}(p \kappa / 2)$ and $L^{p}(p \kappa / 2)$, are imbeddable in $\mathfrak{S}$.

Particularly interesting is the next problem concerning the limiting case $p \rightarrow \infty$.

* The following is a different phrasing of the fact that $L^{p}(p / 2)$ is imbeddable in $L^{2}:$ If $0<p<2$, then there is a functional $y(t)=U[x(t)]$, defined for all functions $x(t)$ in $L^{p}$, with values $y(t)$ in $L^{2}$ such that the relation

$$
\int_{0}^{1}\left|x_{1}(t)-x_{2}(t)\right|^{p} d t=\int_{0}^{1}\left|y_{1}(t)-y_{2}(t)\right|^{2} d t
$$

holds for two arbitrary $x_{1}(t), x_{2}(t)$ of $L^{p}$. This functional $y=U[x]$ is necessarily continuous and univalent. A study of its further properties might prove to be of interest in the theory of $L^{p}$. 
Problem 2. Do there exist positive exponents $\gamma$ such that the function

$$
\exp \left[-\left[\max \left(\left|x_{1}\right|, \cdots,\left|x_{m}\right|\right)\right]^{2 \gamma}\right]=\min \left(e^{-\left|x_{1}\right| 2 \gamma}, \cdots, e^{-\left|x_{m}\right| 2 \gamma}\right)
$$

is positive definite?

Again, if such exponents exist, there would exist a positive number $\gamma_{m}$ with the properties: (23) is p.d. if $0<\gamma \leqq \gamma_{m}$ and is not p.d. if $\gamma>\gamma_{m}$. Moreover the function (23) is p.d. if and only if $E_{m}^{\infty}(\gamma)$ is imbeddable in $\mathfrak{S}$.

The particular interest of this second problem is due to the following lemma which is due essentially to Fréchet ([6], pp. 161-162). Let us call a finite metric set and denote by $\sigma_{m}$ a metric space composed of exactly $m+1$ distinct points.

Lemma. Any finite metric set $\sigma_{m}$ of $m+1$ points may be imbedded isometrically in $E_{m}^{\infty}$.

Proof. Let $P_{0}, P_{1}, \cdots, P_{m}$ denote the points of $\sigma_{m}$ and $\overline{P_{i} P_{k}}$ their distances. Consider in $E_{m}^{\infty}$ the following $m+1$ points:

$Q_{i}=\left(x_{1 i}, x_{2 i}, \cdots, x_{m i}\right) \equiv\left({\overline{P_{1} P_{i}}},{\overline{P_{2} P_{i}}}, \cdots,{\overline{P_{m} P_{i}}}\right), \quad i=0,1, \cdots, m$.

For their distance $\overline{Q_{i} Q_{k}}$ in $E_{m}^{\infty}$ we have

$$
\overline{Q_{i} Q_{k}}=\max _{\mathrm{j}=1, \cdots, m}\left|x_{j i}-x_{j k}\right|=\max _{\mathrm{j}=1, \cdots, m}\left|\overline{P_{j} P_{i}}-\overline{P_{j} P_{k}}\right|=\overline{P_{i} P_{k}},
$$

for certainly $\left|\overline{P_{j} P_{i}}-\overline{P_{j} P_{k}}\right| \leqq \overline{P_{i} P_{k}},(j=1, \cdots, m)$, on account of the triangle inequality in $\sigma_{m}$, while the equality sign holds if $j$ is equal to whichever of the numbers $i$ or $k$ happens to be different from zero and hence within the range of $j$. If both $i=k=0$, the result $\overline{Q_{0} Q_{0}}=0$ was clear from the start.

On the basis of this lemma it is natural to classify finite metric sets according to their "dimension" as follows: $A$ set $\sigma_{n}$ is said to be of dimension $m$ (always less than or equal to $n$, on account of the lemma) if it is imbeddable in $E_{m}^{\infty}$, but not in $E_{m-1}^{\infty}$. If the last requirement is removed, the dimension of $\sigma_{n}$ does not exceed $m$.

If Problem 2 were solved in the affirmative, we would get the following statement: If $\sigma_{n}$ is any finite metric set of dimension less than or equal to $m$, then $\sigma_{n}(\gamma)$ is imbeddable in $E_{n}$ if $0<\gamma \leqq \gamma_{m}$, and $\gamma_{m}$ is the best constant.

This would generalize in a certain sense the following theorem due to L. M. Blumenthal ([2], p. 402): If $\sigma_{3}$ is a finite metric set composed of four points, then $\sigma_{3}(\gamma)$ is imbeddable in $E_{3}$ if $0<\gamma \leqq 1 / 2$, and $1 / 2$ is the best constant.

In conclusion let us point out the following perhaps not trivial remark concerning Problem 2: $\gamma_{2}$ exists and is greater than or equal to $1 / 2$. Indeed, in view of the formula 


$$
e^{-|t|}=\frac{1}{\pi} \int_{-\infty}^{\infty} \frac{e^{i t x}}{1+x^{2}} d x
$$

we get by the transformation of variables $u=(\xi+\eta) / 2, v=(\xi-\eta) / 2$ in the double integral

$$
\begin{aligned}
\int_{-\infty}^{\infty} & \int_{-\infty}^{\infty} \exp [i(x u+y v)] \frac{d u d v}{\left[1+(u+v)^{2}\right]\left[1+(u-v)^{2}\right]} \\
& =\frac{1}{2} \int_{-\infty}^{\infty} \exp [i \xi(x+y) / 2] \frac{d \xi}{1+\xi^{2}} \cdot \int_{-\infty}^{\infty} \exp [i \eta(x-y) / 2] \frac{d \eta}{1+\eta^{2}} \\
& =\frac{\pi^{2}}{2} \exp \left[-\frac{1}{2}|x+y|-\frac{1}{2}|x-y|\right]=\frac{\pi^{2}}{2} \exp [-\max (|x|,|y|)],
\end{aligned}
$$

due to the relation $2 \max (|x|,|y|)=|x+y|+|x-y|$. This formula shows that the function (23) is p.d. if $m=2, \gamma=1 / 2$. Hence this much is proved:

If $\sigma_{n}$ is any finite metric set of dimension not exceeding 2, then $\sigma_{n}(\gamma)$, $(0<\gamma \leqq 1 / 2)$, is imbeddable in $E_{n}$.

If $\gamma_{3}$ exists, then it is certainly less than or equal to $1 / 2$, by Blumenthal's theorem, and is probably even less than $1 / 2$. A certain special set $\sigma_{4}$ shows that if $\gamma_{4}$ exists, it must be less than 0.45 .

\section{BIBLIOGRAPHY}

1. S. Banach, Théorie des Opérations Linéaires, Warsaw, 1932.

2. L. M. Blumenthal, New theorems and methods in determinant theory, Duke Mathematical Journal, vol. 2 (1936), pp. 396-404.

3. S. Bochner, Vorlesungen über Fouriersche Integrale, Leipzig, 1932.

4. - Monotone Funktionen, Stieltjessche Integrale und harmonische Analyse, Mathematische Annalen, vol. 108 (1933), pp. 378-410.

5. - Stable laws of probability and completely monotone functions, Duke Mathematical Journal, vol. 3 (1937), pp. 726-728.

6. M. Fréchet, Les dimensions d'un ensemble abstrait, Mathematische Annalen, vol. 68 (1910), pp. $145-168$.

7. G. H. Hardy, J. E. Littlewood, and G. Pólya, Inequalities, Cambridge, 1934.

8. J. von Neumann and I. J. Schoenberg, Fourier integrals and metric geometry, to appear in the Transactions of the American Mathematical Society.

9. G. Pólya and G. Szegö, Aufgaben und Lehrsätze aus der Analysis, vol. 2, Berlin, 1925.

10. I. J. Schoenberg, On certain metric spaces arising from euclidean spaces by a change of metric and their imbedding in Hilbert space, Annals of Mathematics, (2), vol. 38 (1937), pp. 787-793.

11. I. Schur, Bemerkungen zur Theorie der beschränkten Bilinearformen mit unendlich vielen Veränderlichen, Journal für die reine und angewandte Mathematik, vol. 140 (1911), pp. 1-28.

12. W. A. Wilson, On certain types of continuous transformations of metric spaces, American Journal of Mathematics, vol. 57 (1935), pp. 62-68.

Colby College,

WATERVILle, ME. 